\title{
The Hellan-Herrmann-Johnson Method: Some New Error Estimates and Postprocessing
}

\author{
By M. I. Comodi*
}

\begin{abstract}
We analyze the behavior of the mixed Hellan-Herrmann-Johnson method for solving the biharmonic problem $\Delta^{2} \psi=f$. We show a superconvergence result for the distance between $\psi^{h}$ (the approximation of the displacement) and $P_{h} \psi$ (where $P_{h}$ is a suitable projection operator). If the discrete equations are solved (as is usually done) by using interelement Lagrange multipliers, our superconvergence result allows us to prove the convergence, in suitable norms, of the Lagrange multipliers to the normal derivative of the displacement, and to construct a new approximation of $\nabla \psi$ which converges to $\nabla \psi$ faster than $\nabla \psi^{h}$.
\end{abstract}

1. Introduction. Many authors, in the last few years, have studied from a theoretical and applied point of view (e.g., [7], [9], [10], [20]) the Hellan-HerrmannJohnson (HHJ) mixed finite element method [13], [14], [15], [16] for the solution of fourth-order linear elliptic boundary value problems of the following type: given $f \in L^{2}(\Omega)$,

$$
\left\{\begin{array}{l}
\text { find } w \in H_{0}^{2}(\Omega) \text { such that } \\
\Delta^{2} w=f \quad \text { in } \Omega .
\end{array}\right.
$$

The HHJ scheme is based on a splitting of the problem (1.1) in two second-order equations in the unknowns $(\psi, \mathbf{u})$, where $\psi=w$ and $u_{i j}=\partial^{2} w / \partial x_{i} \partial x_{j}$. The only drawback is that it leads (as the mixed schemes usually do) to the resolution of a linear system with indefinite matrix. This is due to the continuity constraints imposed on the "stress field" $\mathbf{u}\left(u_{i j}=\partial^{2} w / \partial x_{i} \partial x_{j}\right)$. For this reason, Lagrange multipliers, defined at the interelement boundaries, are often introduced as new unknowns in order to obtain the required continuity without imposing it a priori [12]. This results in a new system of equations in the unknowns $\left(\tilde{\mathbf{u}}^{h}, \tilde{\psi}^{h}, \lambda_{h}\right)$ (respectively: stress field, displacement, Lagrange multipliers) that are related to the solution $\left(\mathbf{u}^{h}, \psi^{h}\right)$ of the original system by the equations

$$
\tilde{\mathbf{u}}^{h}=\mathbf{u}^{h}, \quad \tilde{\psi}^{h}=\psi^{h} .
$$

However, in this new system it is very easy to solve a priori for $\tilde{\mathbf{u}}^{h}$ in terms of $\tilde{\psi}^{h}$ and $\lambda_{h}$. The resulting system in the unknowns $\left(\tilde{\psi}^{h}, \lambda_{h}\right)$ now has a symmetric and positive definite matrix, and therefore many numerical methods become available for its solution.

In view of the relations (1.2), the estimates for $\mathbf{u}-\mathbf{u}^{h}$ and $\psi-\psi^{h}$, proved for instance in [16], [7], [3], can still be used for the new system. On the other hand,

Received November 20, 1986; revised September 23, 1987 and February 25, 1988.

1980 Mathematics Subject Classification (1985 Revision). Primary 65N30.

*This work has been partially supported by the M.P.I. (Italy), by the G.N.I.M. of C.N.R. (Italy) and by the I.A.N. of C.N.R. of Pavia (Italy). 
we have now computed a new approximation, $\lambda_{h}$, that we would like to interpret. From the physical point of view, $\lambda_{h}$ represents the rotations in the normal direction at each interelement boundary $\left(\lambda_{h} \simeq \partial w / \partial n\right)$ which are often used by engineers. However, a precise estimate of the error $\left\|\lambda_{h}-\partial w / \partial n\right\|$ (in some suitable norm) was still lacking.

A similar problem has been solved in the case of second-order problems ([2], [5]): the authors prove both the convergence of the Lagrange multipliers and the possibility of improving the error bounds obtained for the original scheme by suitably exploiting the information provided by the new unknowns $\lambda_{h}$.

Arnold and Brezzi [2] have analyzed the Hellan-Herrmann-Johnson scheme in the case $k=1$ ( $k$ is the local degree of $\psi^{h}$ ) with a technique which cannot be generalized: they show that the method, for $k=1$, is equivalent to a slight modification of the Morley method [18].

In the present paper we first analyze the HHJ scheme itself and we show a superconvergence result for the error $\left\|\psi^{h}-P_{h} \psi\right\|_{1}$, where $P_{h}$ is a suitable projection operator. The new estimate is of the same nature as the one proved for secondorder problems in [11]. Then we use the new estimate in order to obtain error bounds for the Lagrange multipliers in suitable norms defined at the interelement boundaries; finally we exploit the above results for constructing, by means of a suitable postprocessing, a new approximation of $\nabla \psi$ which converges with higher order than $\nabla \psi^{h}$. This last estimate is particularly interesting whenever the scheme is applied to solve Stokes problems, where, as it is well known, the main interest is in the velocity field.

An outline of the paper is as follows. In Section 2, for the reader's convenience, we define the notations which are used in the paper. In Section 3 we recall the classical result concerning the HHJ scheme, [3], [13], [14], [15] [16], [7]. Section 4 is devoted to studying the superconvergence result and the error bounds of the Lagrange multipliers. Finally, Section 5 deals with the construction of the new approximation for $\nabla \psi$ with related error estimates.

2. Notations and Green's Formulae. Given a convex polygon $D$ of $\mathbf{R}^{2}$ with boundary $\partial D$ and vertices $a_{i}\left(i=1,2, \ldots, N_{D}\right)$, a scalar-valued function $w$, and a tensor-valued function $\mathbf{v}=\left(v_{i j}\right), i, j=1,2$, we denote (using the summation convention of repeated indices) by $\mathbf{n}=\left(n_{1}, n_{2}\right)$ the unit outward normal to $\partial D$ and by $\mathbf{t}=\left(t_{1}, t_{2}\right)=\left(-n_{2}, n_{1}\right)$ the unit tangent to $\partial D$. We also set

$$
\begin{aligned}
& w_{/ i}=\partial w / \partial x_{i}(i=1,2), \\
& w_{/ n}=w_{/ i} n_{i} \\
& w_{/ t}=w_{/ i} t_{i} \\
& M_{n}(\mathbf{v})=v_{i j} n_{i} n_{j} \\
& M_{n t}(\mathbf{v})=v_{i j} n_{i} t_{j} \\
& Q_{n}(\mathbf{v})=v_{i j / i} n_{j} \\
& K_{n}(\mathbf{v})=\partial M_{n t} / \partial t+Q_{n}(\mathbf{v}), \\
& S_{i}(\mathbf{v})=\text { jumps of } M_{n t}(\mathbf{v}) \text { at the corners } a_{i} \text { of } D, i=1,2, \ldots, N_{D} .
\end{aligned}
$$

Moreover, $|\cdot|_{m, D} \equiv|\cdot|_{m}$ and $\|\cdot\|_{m, D} \equiv\|\cdot\|_{m}$ will denote the usual seminorm and norm in the space $H^{m}(D)=W^{m, 2}(D)$. We refer, for instance, to [1], [17] for the definition of the Sobolev spaces used in this paper. The following Green's formulae 
hold for any $w \in H^{2}(D)$ and any $\mathbf{v} \in\left(H^{2}(D)\right)^{4}$ such that $v_{12}=v_{21}$,

$$
\begin{aligned}
\int_{D} v_{i j} w_{/ i j} d \mathbf{x}= & -\int_{D} v_{i j / i} w_{/ j} d x+\int_{\partial D} M_{n}(\mathbf{v}) w_{/ n} d s \\
& +\int_{\partial D} M_{n t}(\mathbf{v}) w_{/ t} d s \\
\int_{D} v_{i j / i} w_{/ j} d \mathbf{x}= & -\int_{D} v_{i j / i j} w d \mathbf{x}+\int_{\partial D} Q_{n}(\mathbf{v}) w d s \\
\int_{\partial D} M_{n t}(\mathbf{v}) w_{/ t} d s= & -\int_{\partial D} \frac{\partial M_{n t}(\mathbf{v})}{\partial t} w d s+\sum_{i=1}^{N_{D}} S_{i}(\mathbf{v}) w\left(a_{i}\right) .
\end{aligned}
$$

Then, by combining (2.1)-(2.3),

$$
\begin{aligned}
\int_{D} v_{i j} w_{/ i j} d \mathbf{x}= & \int_{D} v_{i j / i j} w d \mathbf{x}+\int_{\partial D} M_{n}(\mathbf{v}) w_{/ n} d s \\
& -\int_{\partial D} K_{n}(\mathbf{v}) w d s+\sum_{i=1}^{N_{D}} S_{i}(\mathbf{v}) w\left(a_{i}\right)
\end{aligned}
$$

In what follows we shall use the notations:

$\mathbf{P}^{k}(D)$ for polynomials of degree $\leq k$ on $D$;

$C$ or $C_{i}, i \in \mathbf{N}$, for positive constants independent of the data and of the decomposition of $D$, generally with different values at different occurences.

3. The Hellan-Herrmann-Johnson Scheme. In this section we recall the well-known mixed formulation of the model problem (1.1). This formulation allows us to approximate the problem by means of the Hellan-Herrmann-Johnson scheme. Moreover, we shall recall the main results concerning the convergence of this discrete scheme, the implementational technique generally applied and the reasons for choosing this technique.

More precisely, let us consider a convex polygon $\Omega$ of $\mathbf{R}^{2}$, with boundary $\partial \Omega$, and let $\mathbf{T}_{h}$ be a decomposition of $\Omega$ into regular triangles $T$, with boundary $\partial T$ and sides $e_{i}, i=1,2,3$, whose maximum diameter is less than $h$. We now introduce the mixed continuous formulation of the model problem (1.1) that allows the application of the Hellan-Herrmann-Johnson method. For this purpose, we consider the following spaces and the following bilinear forms:

-The space

$$
\begin{array}{r}
V=\left\{\mathbf{v}=\left(v_{i j}\right), i, j=1,2, v_{12}=v_{21}, v_{i j} \in L^{2}(\Omega)\right. \\
\left.v_{\left.i j\right|_{T}} \in H^{1}(T) \text { for all } T \in \mathbf{T}_{h}, M_{n}(\mathbf{v}) \text { is c.i.b. }\right\} .
\end{array}
$$

$M_{n}(\mathbf{v})$ is c.i.b. (continuous at the interelement boundaries) if and only if, for any pair $\left(T_{1}, T_{2}\right)$ of adjacent triangles, we have

$$
M_{\mathbf{n}}\left(\mathbf{v}_{\mid T_{1}}\right)=M_{\mathbf{n}}\left(\mathbf{v}_{\mid T_{2}}\right) \quad \text { on } T_{1} \cap T_{2},
$$

where $\mathbf{n}$ is the unit normal to $T_{1} \cap T_{2}$.

A function $\mathbf{v} \in V$ has the following norm

$$
\|\mathbf{v}\|_{V}^{2}=\sum_{T \in \mathbf{T}_{h}}\|\mathbf{v}\|_{1, T}^{2} .
$$


-The finite-dimensional subspace of $V$,

$$
V_{h}=\left\{\mathbf{v}^{h} \in V, v_{i j_{1} T}^{h} \in \mathbf{P}_{k-1}(T) \text { for all } T \in \mathbf{T}_{h}\right\} ;
$$

-the space

$$
W=W_{0}^{1, p}, \quad p>2 ;
$$

-the finite-dimensional subspace of $W$,

$$
W_{h}=\left\{\phi^{h} \in W, \phi_{\left.\right|_{T}}^{h} \in \mathbf{P}_{k}(T) \text { for all } T \in \mathbf{T}_{h}\right\} ;
$$

-the symmetric continuous bilinear form

$$
a(\mathbf{u}, \mathbf{v})=\int_{\Omega} \mathbf{u}_{i j} \mathbf{v}_{i j} d \mathbf{x}, \quad \mathbf{u}, \mathbf{v} \in V
$$

-the continuous bilinear form

$$
b(\mathbf{v}, \phi)=\sum_{T \in \mathbf{T}_{h}}\left(\int_{T} v_{i j / i} \phi_{/ j} d \mathbf{x}-\int_{\partial T} M_{n t}(\mathbf{v}) \phi_{/ t} d s\right), \quad \mathbf{v} \in V, \phi \in W .
$$

Then, with these notations, the mixed formulation of $(1.1)$ is:

$$
\left\{\begin{array}{l}
\text { find }(\mathbf{u}, \psi) \in V \times W \text { such that } \\
a(\mathbf{u}, \mathbf{v})+b(\mathbf{v}, \psi)=0 \quad \text { for all } \mathbf{v} \in V \\
b(\mathbf{u}, \phi)=\int_{\Omega} f \phi d \mathbf{x} \quad \text { for all } \phi \in W
\end{array}\right.
$$

and its numerical approximation, by means of the Hellan-Herrmann-Johnson scheme, is:

$$
\left\{\begin{array}{l}
\text { find }\left(\mathbf{u}^{h}, \psi^{h}\right) \in V_{h} \times W_{h} \text { such that } \\
a\left(\mathbf{u}^{h}, \mathbf{v}^{h}\right)+b\left(\mathbf{v}^{h}, \psi^{h}\right)=0 \quad \text { for all } \mathbf{v}^{h} \in V_{h}, \\
b\left(\mathbf{u}^{h}, \phi^{h}\right)=\int_{\Omega} f \phi^{h} d \mathbf{x} \quad \text { for all } \phi^{h} \in W_{h} .
\end{array}\right.
$$

It is well known that both problems (3.8) and (3.9) have a unique solution $(\mathbf{u}, \psi)$ and $\left(\mathbf{u}^{h}, \psi^{h}\right)$, respectively, where $(\mathbf{u}, \psi)$ is related to the solution of the model problem (1.1) in the following way: $u_{i j}=w_{/ i j}, i, j=1,2 ; \psi=w$.

Many authors have contributed to the mathematical study of this method ([3], [7], [13], [14], [15], [16]). We recall in the following propositions some basic results in approximation theory and in error estimates that will play a fundamental role in our study.

PROPOSITION 3.1. Define the operator $\pi_{h} \in L\left(V, V_{h}\right)$ in the following way: given $v \in V$, for every edge $e$ and for every triangle $T$ of the triangulation $\mathbf{T}_{h}$,

$$
\begin{array}{ll}
\int_{e} M_{n}\left(\mathbf{v}-\pi_{h} \mathbf{v}\right) q_{k-1} d s=0 & \text { for all } q_{k-1} \in \mathbf{P}_{k-1}(e), \\
\int_{T}\left(v_{i j}-\pi_{h} v_{i j}\right) q_{k-2} d \mathbf{x}=0 & \text { for all } q_{k-2} \in \mathbf{P}_{k-2}(T) .
\end{array}
$$

Then

$$
b\left(\mathbf{v}-\pi_{h} \mathbf{v}, \phi^{h}\right)=0 \quad \text { for all } \phi_{h} \in W_{h} .
$$


Moreover, if $\mathbf{v} \in V \cap\left[H^{k}(\Omega)\right]^{4}$,

$$
\left\|\mathbf{v}-\pi_{h} \mathbf{v}\right\|_{0} \leq C h^{k}|\mathbf{v}|_{k}
$$

PROPOSITION 3.2. Define the operator $P_{h} \in L\left(W, W_{h}\right)$ in the following way: given $\phi \in W$, for every vertex $a$, for every edge $e$, and for every triangle $T$ of the triangulation $\mathbf{T}_{h}$,

$$
\begin{aligned}
P_{h} \phi(a)=\phi(a), & \text { for all } q_{k-2} \in \mathbf{P}_{k-2}(e), \\
\int_{\cdot e}\left(\phi-P_{h} \phi\right) q_{k-2} d s=0 & \text { for all } q_{k-3} \in \mathbf{P}_{k-3}(T) . \\
\int_{T}\left(\phi-P_{h} \phi\right) q_{k-3} d \mathbf{x}=0 &
\end{aligned}
$$

Then

$$
b\left(\mathbf{v}^{h}, \phi-P_{h} \phi\right)=0 \quad \text { for all } \mathbf{v}^{h} \in V_{h} .
$$

Moreover, if $\phi \in W \cap H^{k+1}(\Omega)$,

$$
\left\|\phi-P_{h} \phi\right\|_{0} \leq C h^{k+1}|\phi|_{k+1} .
$$

PROPOSITION 3.3. Let $(\mathbf{u}, \psi)$ be the solution of $(3.8),\left(\mathbf{u}^{h}, \psi^{h}\right)$ the solution of (3.9), and let $w \in H^{k+2}(\Omega) \cap H_{0}^{2}(\Omega)$ be the solution of (1.1); then

$$
\left\|\mathbf{u}-\mathbf{u}^{h}\right\|_{0}+\left\|\psi-\psi^{h}\right\|_{1} \leq C h^{k}\left(|w|_{k+1}+|w|_{k+2}\right) .
$$

Moreover,

$$
\left\|\psi-\psi^{h}\right\|_{0} \leq C h^{k+1}\left(|w|_{k+1}+|w|_{k+2}\right)
$$

We now come to the first topic of this paper. The matrix arising from the scheme (3.9) is indefinite. To circumvent this inconvenience, the continuity constraint on the bending moment $M_{n}\left(\mathbf{v}^{h}\right)$ is eliminated from the space $V_{h}$, and new unknowns (Lagrange multipliers), defined at the interelement boundaries, are introduced [12]. For this purpose, we introduce:

-the set

$$
E_{h}=\text { set of the internal edges } e \text { of } \mathbf{T}_{h}
$$

-the spaces

$$
\tilde{V}_{h}=\left\{\mathbf{v}^{h}=\left(v_{i j}\right), i, j=1,2, v_{12}^{h}=v_{21}^{h}, v_{\left.i j\right|_{T}}^{h} \in \mathbf{P}_{k-1}(T)\right.
$$

for all $\left.T \in \mathbf{T}_{h}\right\}$,

-the continuous bilinear form

$$
c\left(\mathbf{v}^{h}, \mu_{h}\right)=\sum_{T \in \mathbf{T}_{n}^{T}} \int_{\partial T} M_{n}\left(\mathbf{v}^{h}\right) \mu_{h} d s, \quad \mathbf{v}^{h} \in \tilde{V}_{h}, \mu_{h} \in M_{h} .
$$

Now consider the following problem:

$$
\begin{cases}\text { find }\left(\tilde{u}^{h}, \tilde{\psi}^{h}, \lambda_{h}\right) \in \tilde{V}_{h} \times W_{h} \times M_{h} & \text { such that } \\ a\left(\tilde{\mathbf{u}}^{h}, \mathbf{v}^{h}\right)+b\left(\mathbf{v}^{h}, \tilde{\psi}^{h}\right)=c\left(\mathbf{v}^{h}, \lambda_{h}\right) & \text { for all } \mathbf{v}^{h} \in \tilde{V}_{h}, \\ b\left(\tilde{u}^{h}, \phi^{h}\right)=\int_{\Omega} f \phi^{h} d \mathbf{x} & \text { for all } \phi^{h} \in W_{h}, \\ c\left(\tilde{\mathbf{u}}^{h}, \mu_{h}\right)=0 & \text { for all } \mu_{h} \in M_{h} .\end{cases}
$$

It is easy to prove the following proposition. 
PROPOSITION 3.4. Problem (3.25) has a unique solution $\left(\tilde{\mathbf{u}}^{h}, \tilde{\psi}^{h}, \lambda_{h}\right)$ such that $\tilde{\mathbf{u}}^{h}=\mathbf{u}^{h}$ and $\tilde{\psi}^{h}=\psi^{h}$, where $\left(\mathbf{u}^{h}, \psi^{h}\right)$ is the solution of (3.9).

We shall identify, then, $\tilde{\mathbf{u}}^{h}$ with $\mathbf{u}^{h}$ and $\tilde{\psi}^{h}$ with $\psi^{h}$. On the other hand, $\lambda_{h}$ clearly approaches $\psi_{/ n}$ at the interelement boundaries. We wish to find an error bound, in some suitable norm, for this approximation. This will be the topic of the next section.

4. Error Estimates for the Lagrangian Multipliers. In [2], results similar to those we want to obtain are proved for second-order elliptic problems; the authors of [2] also treat the Hellan-Herrmann-Johnson scheme, but only in the case $k=1$ and with a technique which does not work for $k \geq 2$. In this section we shall employ the "strategy" used in [2], for second-order elliptic problems, in order to obtain estimates on (3.25) for $k \geq 2$. To do this, we first have to extend the techniques described in [11], [5] for second-order elliptic problems. In particular, we shall consider duality with respect to $H^{r}(\Omega)$ instead of $H_{0}^{r}(\Omega)$.

Definition 4.1. Given $\chi \in L^{2}(\Omega), \chi \not \equiv 0$, we set

$$
\|\chi\|_{-r}=\sup _{\substack{\rho \in H^{r}(\Omega) \\ \rho \neq 0}} \frac{(\rho, \chi)}{\|\rho\|_{r}} .
$$

Moreover, we shall consider the error equations obtained from (3.8), (3.9) and from Proposition 3.2 with

$$
\begin{gathered}
\mathbf{z}=\mathbf{u}-\mathbf{u}^{h}, \\
\xi=P_{h} \psi-\psi^{h} .
\end{gathered}
$$

We have

$$
\begin{gathered}
a\left(\mathbf{z}, \mathbf{v}^{h}\right)+b\left(\mathbf{v}^{h}, \xi\right)=0 \quad \text { for all } \mathbf{v} \in V_{h}, \\
b\left(\mathbf{z}, \phi^{h}\right)=0 \quad \text { for all } \phi^{h} \in W_{h} .
\end{gathered}
$$

We can prove the following lemma.

LEMMA 4.1. Let $\mathrm{z} \in V$ and $\xi \in W_{h}$ be given by (4.2) and (4.3). Then for $-2 \leq r \leq-1+\varepsilon$ (where $\varepsilon$ depends on the maximum angle of $\Omega$ )

$$
\|\xi\|_{-r} \leq C\left(h^{\min (r+2, k)}\|\mathbf{z}\|_{0}+h^{\min (r+3, k)}\left\|\mathbf{u}-\pi_{h} \mathbf{u}\right\|_{1}\right) .
$$

Proof. Let $\chi$ be the solution of the problem

$$
\begin{cases}\Delta^{2} \chi=\rho & \text { in } \Omega, \\ \chi=\chi / n=0 & \text { on } \partial \Omega .\end{cases}
$$

Since $\Omega$ is a convex polygon, we have

$$
\|\chi\|_{r+4} \leq C\|\rho\|_{r} .
$$

With the solution $\chi \in H^{r+4} \cap H_{0}^{2}$ of (4.7) we associate a tensor-valued function $\mathbf{v}$ defined by

$$
v_{i j}=\chi_{/ i j}, \quad i, j=1,2
$$


and we consider the equations (4.4) and (4.5). By Green's formula, the orthogonality (3.12), and by Proposition 3.2 we get

$$
\begin{gathered}
(\rho, \xi)=-b(\mathbf{v}, \xi)=-b\left(\pi_{h} \mathbf{v}, \xi\right)=a\left(\mathbf{z}, \pi_{h} \mathbf{v}\right) \\
a(\mathbf{z}, \mathbf{v})=-b(\mathbf{z}, \chi)=-b\left(\mathbf{z}, \chi-P_{h} \chi\right)
\end{gathered}
$$

that is,

$$
\begin{aligned}
(\rho, \xi) & =a\left(\mathbf{z}, \pi_{h} \mathbf{v}-\mathbf{v}\right)+b\left(\mathbf{z}, \chi-P_{h} \chi\right) \\
& =a\left(z, \pi_{h} \mathbf{v}-\mathbf{v}\right)+b\left(\mathbf{u}-\pi_{h} \mathbf{u}, \chi-P_{h} \chi\right) .
\end{aligned}
$$

By virtue of the definition of the form $b(\cdot, \cdot),(4.2),(3.15)$, and the regularity of $\mathbf{u}$, we have

$$
b\left(\mathbf{u}-\pi_{h} \mathbf{u}, \chi-P_{h} \chi\right)=\sum_{T \in \mathbf{T}_{h}} \int_{T}\left(u_{i j}-\pi_{h} u_{i j}\right)_{/ i}\left(\chi-P_{h} \chi\right)_{/ j} d x
$$

so that

$$
|(\rho, \xi)| \leq C\left(\|\mathbf{z}\|_{0}\left\|\pi_{h} \mathbf{v}-\mathbf{v}\right\|_{0}+\left\|\mathbf{u}-\pi_{h} \mathbf{u}\right\|_{1}\left\|\chi-P_{h} \chi\right\|_{1}\right)
$$

from which, using (3.13), (3.18), (4.8), (4.9) and (4.1), the estimate (4.6) follows.

Remark. The estimate (4.6) will be useful if $k \geq 2$; if $k=1$ it is more useful to obtain a slightly different estimate. Actually, the term $b\left(\mathbf{u}-\pi_{h} \mathbf{u}, \chi-P_{h} \chi\right)$ which appears in (4.12) can easily be estimated as $\left|b\left(\mathbf{u}-\pi_{h} \mathbf{u}, \chi-P_{h} \chi\right)\right| \leq\|f\|_{0}\left\|\chi-P_{h} \chi\right\|_{0}$. By using the latter formula we can obtain

$$
\|\xi\|_{1} \leq C\left(h\|z\|_{0}+h^{2}\|f\|_{0}\right) .
$$

Thanks to the previous lemma we can now give an estimate which will be essential both for measuring the error in the Lagrange multipliers and in postprocessing.

THEOREM 4.2. Let $\psi$ and $\psi^{h}$ be the solutions of (3.8) and (3.9), respectively, and let $P_{h} \psi$ be given by Proposition 3.2. Then

$$
\left\|P_{h} \psi-\psi^{h}\right\|_{1} \leq C h^{k+1}\left(|w|_{k+1}+|w|_{k+2}+\|f\|_{0} \delta_{k 1}\right) .
$$

Proof. The above estimate follows from (4.2), (4.3) and (3.19) by using (4.6) with $r=-1$, if $k \geq 2$, and (4.14) if $k=1$.

We are now able to bound the error $\lambda_{h}-\psi_{/ n}$ defined on $E_{h}$. Following the development of [2], we first of all define the norms on $M_{h}$ :

$$
\begin{aligned}
\left|\mu_{h}\right|_{0, h}^{2} & =\sum_{e \in E_{h}}\left\|\mu_{h}\right\|_{0, e}^{2}, \\
\left|\mu_{h}\right|_{-1 / 2, h}^{2} & =\sum_{e \in E_{h}}|e|\left\|\mu_{h}\right\|_{0, e}^{2},
\end{aligned}
$$

where $|e|$ is the length of $e$, and we define

$$
Q_{h} \phi=\text { orthogonal projection of } \phi_{\mid E_{h}} \text { onto } M_{h} \text { in the norm (4.15a). }
$$

We can then state the following result. 
THEOREM 4.3. Let $w$ be the solution of (1.1), $(\mathbf{u}, \psi)$ the solution of (3.8), $\left(\mathbf{u}^{h}, \psi^{h}, \lambda_{h}\right)$ the solution of (3.25), and let $P_{h} \psi$ and $Q_{h} \psi_{/ n}$ be given by Proposition 3.2 and (4.16), respectively. Then

$$
\left|\lambda_{h}-Q_{h} \psi_{/ n}\right|_{-1 / 2, h} \leq C h^{k+1}\left(|w|_{k+1}+|w|_{k+2}+\|f\|_{0} \delta_{k 1}\right) .
$$

Proof. The estimate (4.17) is an obvious consequence (see (4.15), Theorem 4.2, and Proposition 3.3) of

$$
\left\|\lambda_{h}-Q_{h} \psi_{/ n}\right\|_{0, e} \leq C\left(h_{T}^{1 / 2}\left\|\left(\mathbf{u}-\mathbf{u}^{h}\right)\right\|_{0, T}+h_{T}^{-1 / 2}\left\|P_{h} \psi-\psi^{h}\right\|_{1, T}\right) .
$$

It thus suffices to prove (4.18).

We consider a triangle $T \in \mathbf{T}_{h}$, with boundary $\partial T$, an edge $e \in \partial T$, and we consider $\mathbf{v} \in \tilde{V}_{h}$ uniquely defined by

$$
\begin{gathered}
M_{n}(\mathbf{v})= \begin{cases}\lambda_{h}-Q_{h} \psi_{/ n} & \text { on } e, \\
0 & \text { on } \partial T \backslash e,\end{cases} \\
\int_{T} v_{i j} q_{k-2} d \mathbf{x}=0 \quad \text { for all } q_{k-2} \in \mathbf{P}_{k-2}(T) .
\end{gathered}
$$

By easy scaling arguments we get

$$
\|\mathbf{v}\|_{0, T} \leq C h_{T}^{1 / 2}\left\|\lambda_{h}-Q_{h} \psi_{/ n}\right\|_{0, e} .
$$

Inserting $\mathbf{v}$ in (3.25a) and using Green's formula, Proposition 3.2, and (4.16), we obtain

$$
\begin{gathered}
\int_{T} u_{i j}^{h} v_{i j} d \mathbf{x}+\int_{T} v_{i j / i} \psi_{/ j}^{h} d \mathbf{x}-\int_{\partial T} M_{n t}(\mathbf{v}) \psi_{/ t}^{h} d s-\int_{e} M_{n}(\mathbf{v}) \lambda_{h} d s=0 \\
\int_{T} u_{i j} v_{i j} d \mathbf{x}+\int_{T} v_{i j / i} \psi_{/ j} d \mathbf{x}-\int_{\partial T} M_{n t}(\mathbf{v}) \psi_{/ t} d s-\int_{e} M_{n}(\mathbf{v}) \psi_{/ n} d s \\
=\int_{T} u_{i j} v_{i j} d \mathbf{x}+\int_{T} v_{i j / i} P_{h} \psi_{/ j} d \mathbf{x}-\int_{\partial T} M_{n t}(\mathbf{v}) P_{h} \psi_{/ t} d s \\
-\int_{e} M_{n}(\mathbf{v}) Q_{h} \psi_{/ n} d s
\end{gathered}
$$

Adding the last equations, the definition (4.19) and, once more, Green's formula, yields

$$
\begin{aligned}
\left\|\lambda_{h}-Q_{h} \psi_{/ n}\right\|_{0, e}^{2}= & \int_{T}\left(u_{i j}^{h}-u_{i j}\right) v_{i j} d \mathbf{x}-\int_{T} v_{i j}\left(\psi^{h}-P_{h} \psi\right)_{/ i j} d \mathbf{x} \\
& +\int_{e}\left(\lambda_{h}-Q_{h} \psi / n\right)\left(\psi^{h}-P_{h} \psi\right)_{/ n} d s .
\end{aligned}
$$

By known trace theorems [19] and by simple scaling arguments we have

$$
\begin{aligned}
& \int_{e}\left(\lambda_{h}-Q_{h} \psi_{/ n}\right)\left(\psi^{h}-P_{h} \psi\right)_{/ n} d s \\
& \quad \leq C\left\|\lambda_{h}-Q_{h} \psi_{/ n}\right\|_{0, e}\left(h_{T}^{-1 / 2}\left|\psi^{h}-P_{h} \psi\right|_{1, T}+h_{T}^{1 / 2}\left|\psi^{h}-P_{h} \psi\right|_{2, T}\right) .
\end{aligned}
$$

Then, by applying (4.21), (4.20) and (4.25) we get (4.18) from (4.24). 
5. Postprocessing of the Hellan-Herrmann-Johnson Scheme. In this section we want to "improve" the approximation of the original unknowns by means of a suitable postprocessing. The problem can be solved by different techniqes (e.g., $[1],[4])$. We shall use the estimates previously proved:

$$
\left\|\psi^{h}-P_{h} \psi\right\|_{1} \leq C h^{k+1}\left(|w|_{k+1}+|w|_{k+2}+\|f\|_{0} \delta_{k 1}\right)
$$

and, for all $e \in E_{h}$,

$$
\left\|\lambda_{h}-Q_{h} \psi_{/ n}\right\|_{0, e} \leq C\left(h_{T}^{1 / 2}\left\|\mathbf{u}-\mathbf{u}^{h}\right\|_{0, T}+h_{T}^{-1 / 2}\left\|\psi^{h}-P_{h} \psi\right\|_{1, T}\right),
$$

in order to find a "better approximation" of $\nabla \psi$.

It is well known that the Hellan-Herrmann-Johnson scheme is mainly applied to either solve plate problems (then $\psi:=$ transversal displacement) or to solve Stokes problems (then $\psi:=$ stream function); an approximation of $\nabla \psi$, which represents the velocity field, will be particularly interesting for Stokes problems. Before defining this "new" approximating function, let us remark upon the orthogonalities arising from the definition of $F_{h} \phi$.

Proposition 5.1. Let $\phi \in W$, and let $P_{h} \phi \in W_{h}$ be defined as in Proposition (3.2). Then, for all $e \in E_{h}$,

$$
\int_{e}\left(\phi-P_{h} \phi\right)_{/ t} q_{k-1} d s=0 \quad \text { for all } q_{k-1} \in \mathbf{P}_{k-1}(e),
$$

and for all $T \in \mathbf{T}_{h}$,

$$
\int_{T}\left(\phi-P_{h} \phi\right)_{/ j} q_{k-2} d s=0, \quad j=1,2 \text { for all } q_{k-2} \in \mathbf{P}_{k-2}(T) .
$$

Proof. Consider a triangle $T \in \mathbf{T}_{h}$, with boundary $\partial T$, and an edge $e$ of $\partial T$. The conditions (3.14) and (3.15) immediately imply (5.3) with $q_{k-2}=q_{k-1 / t}$ (t: unit tangent to $e$ ). Analogously, (3.15) and (3.16) imply (5.4), since

$$
\begin{aligned}
\int_{T}\left(\phi-P_{h} \phi\right)_{/ j} q_{k-2} d \mathbf{x}=- & \int_{T}\left(\phi-P_{h} \phi\right) q_{k-2 / j} d \mathbf{x} \\
& +\int_{\partial T}\left(\phi-P_{h} \phi\right) q_{k-2} n_{j} d s, \quad j=1,2 .
\end{aligned}
$$

Now we shall use $\psi^{h}$ and $\lambda_{h}$, which are respectively a polynomial of degree $\leq k$ in each $T \in \mathbf{T}_{h}$ and a polynomial of degree $\leq k-1$ on each $e \in E_{h}$, in order to define a vector $\tau$ belonging to the following space:

$$
\begin{aligned}
S_{h}=\left\{\boldsymbol{\sigma} \in\left[L^{2}(\Omega)\right], \boldsymbol{\sigma}=\left(\sigma_{j}\right), j=1,2,\right. & \sigma_{\left.j\right|_{T}} \in \mathbf{P}_{k}(T), \\
& \left.j=1,2, \text { for all } T \in \mathbf{T}_{h}\right\},
\end{aligned}
$$

which converges to $\nabla \psi$ faster than $\nabla \psi^{h}$.

Unfortunately, we have to distinguish between the cases $k$ odd and $k$ even, as the technique we shall use for $k$ odd does not work for $k$ even. However, we shall succeed in our task following the strategy suggested in [6].

Let us introduce the following lemma. In this section, the index $i$ will assume the values $1,2,3$, and the index $l$ the values 1 or 2 . 
LEMMA 5.2. Let $k$ be a nonnegative odd integer, and let $T$ be a triangle of $\mathbf{T}_{h}$, with edges $e_{i}$. Then, given $\mu_{n}, \mu_{t} \in L^{2}\left(e_{i}\right)$, and $\phi \in\left[L^{2}(T)\right]^{2}$, a vector $\sigma \in S_{h}$ is uniquely defined on $T$ by the following conditions:

$$
\begin{array}{ll}
\int_{e_{i}}\left(\boldsymbol{\sigma} \mathbf{n}-\mu_{n}\right) q_{k-1} d s=0 & \text { for all } q_{k-1} \in \mathbf{P}_{k-1}\left(e_{i}\right) \\
\int_{e_{i}}\left(\boldsymbol{\sigma t}-\mu_{t}\right) q_{k-1} d s=0 & \text { for all } q_{k-1} \in \mathbf{P}_{k-1}\left(e_{i}\right) \\
\int_{T}\left(\sigma_{l}-\phi_{l}\right) q_{k-3} d \mathbf{x}=0 & \text { for all } q_{k-3} \in \mathbf{P}_{k-3}(T) .
\end{array}
$$

Moreover,

$$
\|\boldsymbol{\sigma}\|_{0, T} \leq C\left(h^{1 / 2} \sum_{1^{i}}^{3}\left(\left\|\mu_{n}\right\|_{0, e_{i}}+\left\|\mu_{t}\right\|_{0, e_{i}}\right)+\|\phi\|_{0, T}\right) .
$$

Proof. The number of equations and unknowns of the system (5.7)-(5.9) is

$$
3 k+3 k+(k-2)(k-1)=(k+1)(k+2),
$$

i.e., the dimension of $S_{h}$; then, to verify that the conditions (5.7)-(5.9) identify $\sigma$ in a unique way, we have only to prove that taking $\mu_{n}=\mu_{t}=\phi_{l}=0$ we get $\boldsymbol{\sigma} \equiv 0$. In this case, the conditions (5.7), (5.8) imply that each component of $\boldsymbol{\sigma}$ is, on each edge, a multiple of a Legendre polynomial of degree $k$. Since $k$ is odd, these polynomials have to take values of opposite signs at the endpoints of each edge; then the continuity of $\sigma_{l}$ yields $\sigma_{l}=0$ on $\partial T$, or $\sigma$ has the form $\sigma_{l}=\lambda_{1} \lambda_{2} \lambda_{3} p_{k-3}^{(l)}$, $p_{k-3}^{(l)} \in \mathbf{P}_{k-3}(T), \lambda_{i}:=$ barycentric coordinates on $T$. Now, (5.9), with $\phi_{l}=0$, $q_{k-3}=p_{k-3}^{(l)}$, immediately gives $\sigma \equiv 0$. A standard scaling argument leads to (5.10).

We are finally able to prove the desired estimate.

THEOREM 5.3. Let $k$ be a nonnegative odd integer,

$$
(\mathbf{u}, \psi) \in\left[H^{k}(\Omega)\right]^{4} \times\left\{H^{k+2}(\Omega) \cap H_{0}^{2}(\Omega)\right\}
$$

the solution of (3.8), $w=\psi$ the solution of (1.1), $\left(\mathbf{u}^{h}, \psi^{h}, \lambda_{h}\right) \in \tilde{V}_{h} \times W_{h} \times M_{h}$ the solution of (3.25), and let $\tau \in S_{h}$ be uniquely defined by the following conditions:

$$
\begin{array}{ll}
\int_{e_{i}}\left(\boldsymbol{\tau} \mathbf{n}-\lambda_{h}\right) q_{k-1} d s=0 & \text { for all } q_{k-1} \in \mathbf{P}_{k-1}\left(e_{i}\right), \\
\int_{e_{i}}\left(\tau \mathbf{t}-\psi_{/ t}^{h}\right) q_{k-1} d s=0 & \text { for all } q_{k-1} \in \mathbf{P}_{k-1}\left(e_{i}\right), \\
\int_{T}\left(\tau_{l}-\psi_{/ l}^{h}\right) q_{k-3} d \mathbf{x}=0 & \text { for all } q_{k-3} \in \mathbf{P}_{k-3}(T) .
\end{array}
$$

Then

$$
\|\tau-\nabla \psi\|_{0, T} \leq C h^{k+1}\left(|w|_{k+1}+|w|_{k+2}+\|f\|_{0} \delta_{k l}\right)
$$


Proof. We define an auxiliary function $\tau^{*} \in S_{h}$ by means of the following equations:

$$
\begin{array}{ll}
\int_{e_{i}}\left(\boldsymbol{\tau}^{*} \mathbf{n}-\psi_{/ n}\right) q_{k-1} d s=0 & \text { for all } q_{k-1} \in \mathbf{P}_{k-1}\left(e_{i}\right) \\
\int_{e_{i}}\left(\tau^{*} \mathbf{t}-\psi_{/ t}\right) q_{k-1} d s=0 & \text { for all } q_{k-1} \in \mathbf{P}_{k-1}\left(e_{i}\right) \\
\int_{T}\left(\tau_{l}^{*}-\psi_{/ l}\right) q_{k-3} d \mathbf{x}=0 & \text { for all } q_{k-3} \in \mathbf{P}_{k-3}(T)
\end{array}
$$

Known arguments of interpolation imply

$$
\left\|\tau^{*}-\nabla \psi\right\|_{0} \leq C h^{k+1}|\psi|_{k+2},
$$

and by (5.12)-(5.14), (5.16)-(5.18), (4.16) and Lemma 5.1 we get for all $q_{k-1} \in$ $\mathbf{P}_{k-1}\left(e_{i}\right)$ and for all $q_{k-3} \in \mathbf{P}_{k-3}(T)$,

$$
\begin{aligned}
\int_{e_{i}}\left(\boldsymbol{\tau} \mathbf{n}-\tau^{*} \mathbf{n}\right) q_{k-1} d s & =\int_{e_{i}}\left(\lambda_{h}-Q_{h} \psi / n\right) q_{k-1} d s \\
\int_{e_{i}}\left(\boldsymbol{\tau} \mathbf{t}-\tau^{*} \mathbf{t}\right) q_{k-1} d s & =\int_{e_{i}}\left(\psi^{h}-P_{h} \psi\right)_{/ t} q_{k-1} d s \\
\int_{T}\left(\tau_{l}-\tau_{l}^{*}\right) q_{k-3} d \mathbf{x} & =\int_{T}\left(\psi^{h}-P_{h} \psi\right)_{/ l} q_{k-3} d \mathbf{x} .
\end{aligned}
$$

This means, by Lemma 5.2 , that

$$
\begin{aligned}
& \left\|\tau-\tau^{*}\right\|_{0, T} \\
& \quad \leq C\left(h^{1 / 2} \sum_{i=1}^{3}\left(\left\|\lambda_{h}-Q_{h} \psi_{/ n}\right\|_{0, e_{i}}+\left|\psi^{h}-P_{h} \psi\right|_{1, e_{i}}\right)+\left|\psi^{h}-P_{h} \psi\right|_{1, T}\right) .
\end{aligned}
$$

Then (5.15) is a simple consequence of the triangle inequality, (5.23), (5.19), (5.2) and (5.1).

Let us now treat the case $k$ even.

As we pointed out, the previous technique no longer works, but following [6], we shall find the desired estimate increasing by one the number of the degrees of freedom (hence also of the unknowns) which determine $\tau$. More specifically: given $L_{k}\left(e_{i}\right)$, the Legendre polynomial of degree $k$ on $e_{i}$, assuming the value 1 at the endpoints of $e_{i}$, we define $\chi_{k+1} \in \mathbf{P}_{k+1}(T)$ and $\gamma_{k} \in \mathbf{P}_{k}(T)$ by the following conditions:

$$
\begin{aligned}
& \chi_{k+1}\left(a_{i}\right)=0, \quad a_{i} \text { the corners of } T, \\
& \chi_{k+1 /\left.t\right|_{e_{i}}}=L_{k}\left(e_{i}\right), \\
& \int_{T} \chi_{k+1} q_{k-2} d \mathbf{x}=0 \text { for all } q_{k-2} \in \mathbf{P}_{k-2}(T), \\
& \gamma_{k}=L_{k}\left(e_{i}\right)=\chi_{k+1 / t} \text { on } \partial T, \\
& \int_{T} \gamma_{k} q_{k-3} d \mathbf{x}=0 \quad \text { for all } q_{k-3} \in \mathbf{P}_{k-3}(T),
\end{aligned}
$$

and we define the space

$$
\tilde{S}_{h}=\left\{\boldsymbol{\sigma} \in\left[L^{2}(\Omega)\right]^{2}, \sigma_{\left.j\right|_{T}} \in \mathbf{P}_{k}(T) \oplus\left\{\chi_{k+1}\right\} \text { for all } T \in \mathbf{T}_{h}\right\} .
$$

We can now define $\tau \in \tilde{S}_{h}$ which satisfies (5.15). 
THEOREM 5.4. Let $k$ be a nonnegative even integer, $(\mathbf{u}, \psi), w$ and $\left(\mathbf{u}^{h}, \psi^{h}, \lambda_{h}\right)$ as in Theorem 5.3, and let $\tau \in \tilde{S}_{h}$ be defined by (5.12)-(5.14) and

$$
\int_{T}\left(\tau_{l}-\psi_{/ l}^{h}\right) \Delta \gamma_{k} d \mathbf{x}=0
$$

Then the error bound (5.15) still holds.

Proof. Since the number of degrees of freedom of $\tau$ is equal to the dimension of $\tilde{S}_{h}$, we have only to show that, taking in (5.12)-(5.14) and (5.30) $\lambda_{h}=\psi_{/ t}^{h}=\psi_{/ l}^{h}=0$, we get $\tau \equiv 0$. Now, if $\tau$ has the form

$$
\tau_{l}=p_{k}^{(l)}+\beta_{l} \chi_{k+1}, \quad \beta_{l} \in \mathbf{R},
$$

(5.12) and (5.13), with $q_{k-1}=\gamma_{k_{/ t}}$, and summing over $i$, imply, owing to (5.24)(5.27), that

$$
\beta_{l} \int_{\partial T} \gamma_{k}^{2} d s=0, \quad \text { i.e., } \beta_{k}=0
$$

and this means that, once more by (5.12) and (5.13), $\tau$ has the form

$$
\tau_{l}=\alpha_{l} \gamma_{k}+\lambda_{1} \lambda_{2} \lambda_{3} p_{k-3}^{(l)}
$$

where $\alpha_{l} \in \mathbf{R}, p_{k-3}^{(l)} \in \mathbf{P}_{k-3}(T)$ and $\lambda_{i}$ are the barycentric coordinates of $T$. Taking in (5.14) $q_{k-3}=p_{k-3}^{(l)}$, we get

$$
\int_{T}\left(\alpha_{l} \gamma_{k}+\lambda_{1} \lambda_{2} \lambda_{3} p_{k-3}^{(l)}\right) p_{k-3}^{(l)} d \mathbf{x}=0
$$

from which, by (5.28), $p_{k-3}^{(l)} \equiv 0$ or

$$
\tau_{l}=\alpha_{l} \gamma_{k}
$$

Then (5.30) and (5.27) give

$$
\int_{T} \alpha_{l} \gamma_{k} \Delta \gamma_{k}=-\alpha_{l}\left|\gamma_{k}\right|_{1, T}^{2}
$$

i.e., $\alpha_{l}=0$.

The same arguments used in Theorem 5.3 complete the proof. In particular, observe that, owing to (5.4), we have

$$
\int_{T}\left(\tau_{l}-\tau_{l}^{*}\right) \Delta \gamma_{k} d \mathbf{x}=\int_{T}\left(\psi^{h}-P_{h} \psi\right)_{/ l} \Delta \gamma_{k} d \mathbf{x}
$$

Dipartimento di Matematica

Università di Pavia

27100 Pavia, Italy

1. R. AdAMS, Sobolev Spaces, Academic Press, New York, 1975.

2. D. N. ARNOLD \& F. BREZZI, "Mixed and nonconforming finite element methods: Implementation, postprocessing and error estimates," RAIRO Modél. Math. Anal. Numér., v. 19, 1985, 7-32.

3. I. BABUŠKA, J. OSBORN \& J. PitKäranta, "Analysis of mixed methods using mesh dependent norms," Math. Comp., v. 35, 1980, pp. 1039-1062. 
4. J. H. BRAMBLE \& J. XU, "A local post-processing technique for improving the accuracy in mixed finite element approximation," SIAM J. Numer. Anal. (To appear.)

5. F. BREzZI, J. DOUglas, JR. \& L. D. MARINI, "Two families of mixed finite elements for second order elliptic problems," Numer. Math., v. 47, 1985, pp. 217-435.

6. F. BREzzI \& M. Fortin, Mixed and Hybrid F.E.M. (To appear.)

7. F. BREzzI \& P. A. RAVIART, "Mixed finite element methods for 4th order elliptic equations," in Topics in Numerical Analysis, Vol. III (J.J.H. Miller, ed.), Academic Press, London, 1977, pp. 33-56.

8. P. G. Ciarlet, The Finite Element Method for Elliptic Problems, North-Holland, Amsterdam, 1978.

9. C. Chinosi, L. Della Croce, L. D. Marini, A. Quarteroni, G. Sacchi \& T. SCAPOLla, Implementation of Some Non Standard Finite Element Method for Fourth Order Problems, Report 231 of I.A.N.-C.N.R., Pavia, 1979.

10. M. I. COMODI, "Approximation of a bending plate problem with a boundary unilateral constraint," Numer. Math., v. 47, 1985, pp. 435-458.

11. J. Douglas, JR. \& J. E. RoberTS, "Global estimates for mixed methods for second order elliptic equations," Math. Comp., v. 44, 1985, pp. 39-52.

12. B. Fraejis De VeUBEKe, "Displacement and equilibrium models in the finite element method," in Stress Analysis (O.C. Zienkiewicz and C. Holister, eds.), Wiley, New York, 1965.

13. K. Hellan, Analysis of Elastic Plates in Flexure by a Simplified Finite Element Method, Acta Polytechnica Scandinavica, Ci 46, Trondheim, 1967.

14. K. HerrmanN, "Finite element bending analysis for plates," J. Eng. Mech. Div. ASCE, a3, EM5 93, 1967, pp. 49-83.

15. L. HerrmanN, A Bending Analysis for Plates, Proc. Conf. on Matrix Methods in Structural Mechanics, AFFDL-TR-66-88, 1965, pp. 577-604.

16. C. JOHNSON, "On the convergence of a mixed finite element method for plate bending problems," Numer. Math., v. 21, 1973, pp. 43-62.

17. J. L. LiOns \& E. MAGEnes, Problèmes aux Limites non Homogènes et Applications, Tome I, Dunod, Paris, 1968.

18. L. S. D. MORLEY, "The triangular equilibrium element in the solution of plate bending problems," Aero. Quart., v. 19, 1968, pp. 149-169.

19. J. NEČAS, Les Méthodes Directes en Théorie des Équations Elliptiques, Academia, Prague, 1967.

20. T. Scapolla, "A mixed finite element method for the biharmonic problem," RAIRO Anal. Numér., v. 14, 1980, pp. 55-79. 